\title{
Effect of Solvent Polarity on Fluoroscence Spectra of Camphor Sulphonic Acid Doped Polyaniline
}

\author{
Lakshmidevi V1, Parvathi $\mathbf{P}^{\mathbf{1}}$ and Venkataraman $\mathbf{A}^{\mathbf{1}, \mathbf{2}^{*}}$ \\ ${ }^{I}$ Materials Chemistry Laboratory, Department of Materials Science, Gulbarga University, Kalaburagi-585106, Karnataka, India \\ 2Department of Chemistry, Gulbarga University, Kalaburagi-585106, Karnataka, India
}

\section{Article Info}

*Corresponding author:
Venkataraman A
Department of Chemistry
Gulbarga University
kalaburagi-585106
Karnataka, India
Email: raman.dms@gmail.com

Received: January 9, 2017

Accepted: January 24, 2017

Published: January 27, 2017

Citation: Lakshmidevi V, Parvathi P, Venkataraman A. Effect of Solvent Polarity on Fluoroscence Spectra of Camphor Sulphonic Acid Doped Polyaniline. Madridge J Anal Sci Instrum. 2017; 2(1): 21-24.

doi: $10.18689 /$ mjai-1000105

Copyright: @ 2017 The Author(s). This work is licensed under a Creative Commons Attribution 4.0 International License, which permits unrestricted use, distribution, and reproduction in any medium, provided the original work is properly cited.

Published by Madridge Publishers

\begin{abstract}
The Camphor sulphonic acid doped polyaniline (CSA-PANi)is synthesized by chemical polymerization method and then characterized by using UV-Visible and FTIR. The surface morphology is studied by SEM and AFM techniques. The degree of crystallinity of PANiis studied by XRD and redox behaviour by cyclic voltametry. Thermal stability of the doped polymer is investigated by TGA and DSC. The fluorescence emission spectra of CSA-PANiare investigated in different solvents such as dimethyl formamide(DMF), dimethyl sulphoxide (DMSO) and N-methyl-2-pyrrolidone (NMP). The fluorescence intensity decreases with increase of solvent polarity. A fluorescence study reveals that the nature (blue or red shift) and amount of the shifts of PANi depends on the solvents used. The significance of present study evolves a trend in solvatochromic shifts of PANi in different solvents.
\end{abstract}

Keywords: Conducting Polymer; PANi; Dipole Moment; Fluorescence; Stokes Shift; Solvatochromic Shift.

\section{Introduction}

Conducting polymers were considered as the futuristic new materials that would lead to the next generation of electronics and optoelectronics devices. Among all conducting polymers PANi and its derivatives have attracted much attention worldwide because of chemical stability, simple preparation and high conductivity, low ionization potential, high electron affinity and the ability to be oxidized or reduced more reversibly than conventional polymer [1-3]. PANi has extended $\pi$-electron delocalization, which is responsible forfluorescence emission and it is a fluorescent conjugated polymer. The photoluminescence of PANiemeraldine salt was reported by Gong etal [4]. The extended conjugation also leads to intermolecular hydrogen bonds between the amine and imine group of the adjacent chain and pi stacking. Hence the polymer chain becomes rigid which induces insolubility and infusibility to the polymer chain. The processability of PANi can be improved by using functionalized dopants like CSA or DBSA which was first reported by Cao. et al [5]. The bulky dopants will reduce the mutual aggregation by increasing the solubility of PANisalt in non-polar and weakly polar solvent.

In recent years considerable effort has been made to design and to synthesize functional molecule that could serve as sensitive sensors for the analytical detection of chemically and biologically important ionic species. Fluorescence sensors have received a considerable attention for their potential applications to biochemical and medical analysis. The effect of solvents on spectral properties of molecules generally referred as solvatochromism has been investigated [6]. The study of solvent effects on fluorophore 
has been the intensive area of research in recent years. Suming Chen et.al. Have studied the effect of local environment the fluorescent probe tyrosine under denaturation conditions [7]. Photophysical properties like Stokes shift and quantum yield are the subject of intensive investigation which have considerable importance in the photophysics and photochemistry. Fluorescenceemission of a molecule is sensitive to environment and solvent polarity which are of significant interest due to their versatile applications in chemistry, biology and environmental science [8].

PANi doped with CSA was synthesized by chemical oxidation method and characterized by different techniques which are reported earlier. In this paper we focus on the investigation of Fluorescence studies of CSA-PANi in different solvents which sheds some light on the effect of polarity on the fluorescence spectra.

\section{Materials and methods}

All chemicals and solvents used for synthesis and fluorescence measurements were of analytical grade and used as received.

Fluorescence measurements were done using Elico SL174 Spectro photometer equipped with a Xenon arc lamp. The slit width for excitation and emission were fixed at $5 \mathrm{~nm}$. All measurements were made using $1 \mathrm{~cm}$ Quartz Cuvette at room temperature.

\section{Polymer Solution Preparation}

5.0 ppm Polymer solution was prepared by dissolving 5.0 $\mathrm{mg}$ CSA- PANi in $1000 \mathrm{ml}$ of solvents.

\section{Fluorescence Measurement}

The effect of solvent polarity on the fluorescence of CSAPANi solution was investigated using fixed concentration of PANi in different solvents of different polarities (viz.DMF, NMP and DMSO. The emission spectra of CSA-PANi are measured in polar solvents of different polarity at room temp. Spectroscopic data such as absorption, and emission wavelength, fluorescence intensity and stokes shift are shown in the Table.1

\begin{tabular}{|c|c|c|c|c|c|c|}
\hline Solvent & $\begin{array}{c}\text { Dipole } \\
\text { moment } \\
(\text { Debye) }\end{array}$ & $\begin{array}{c}\text { Excitation } \\
\text { wavelength } \\
(\mathrm{nm})\end{array}$ & $\begin{array}{c}\text { Intensity } \\
(\mathrm{Au})\end{array}$ & $\begin{array}{c}\text { Emission } \\
\text { wavelength } \\
(\mathrm{nm})\end{array}$ & $\begin{array}{c}\text { Intensity } \\
(\mathrm{Au})\end{array}$ & $\begin{array}{c}\text { Stokes } \\
\text { shift(nm) }\end{array}$ \\
\hline DMF & 12.7 & 378 & 674.08 & 409 & 486 & 31 \\
\hline DMSO & 13.5 & 379 & 253 & 408 & 272 & 29 \\
\hline NMP & 13.6 & 374 & 390.42 & 418 & 112 & 37 \\
\hline
\end{tabular}

Table 1: Dipole moment, Excitation wavelength, Emission wavelength and Stokes shift of CSA- PANi in different solvents.

The emission spectra of the polymers solutions were mirror images of the excitation spectra as shown in the figure-1
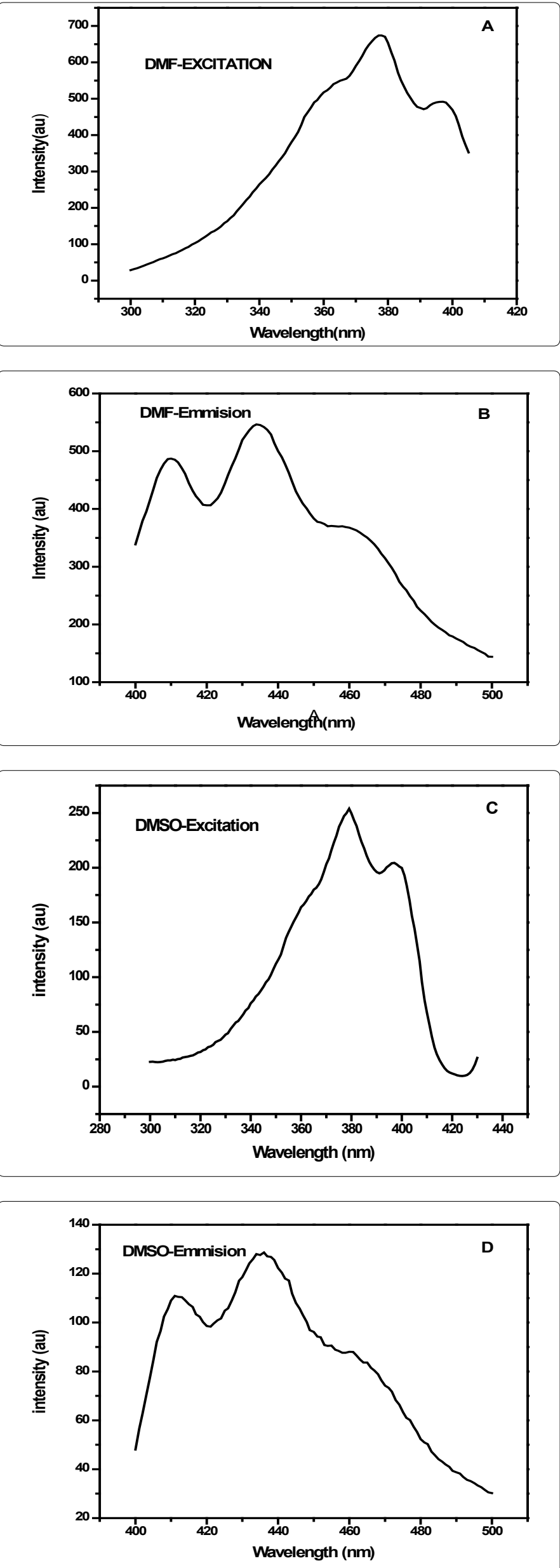

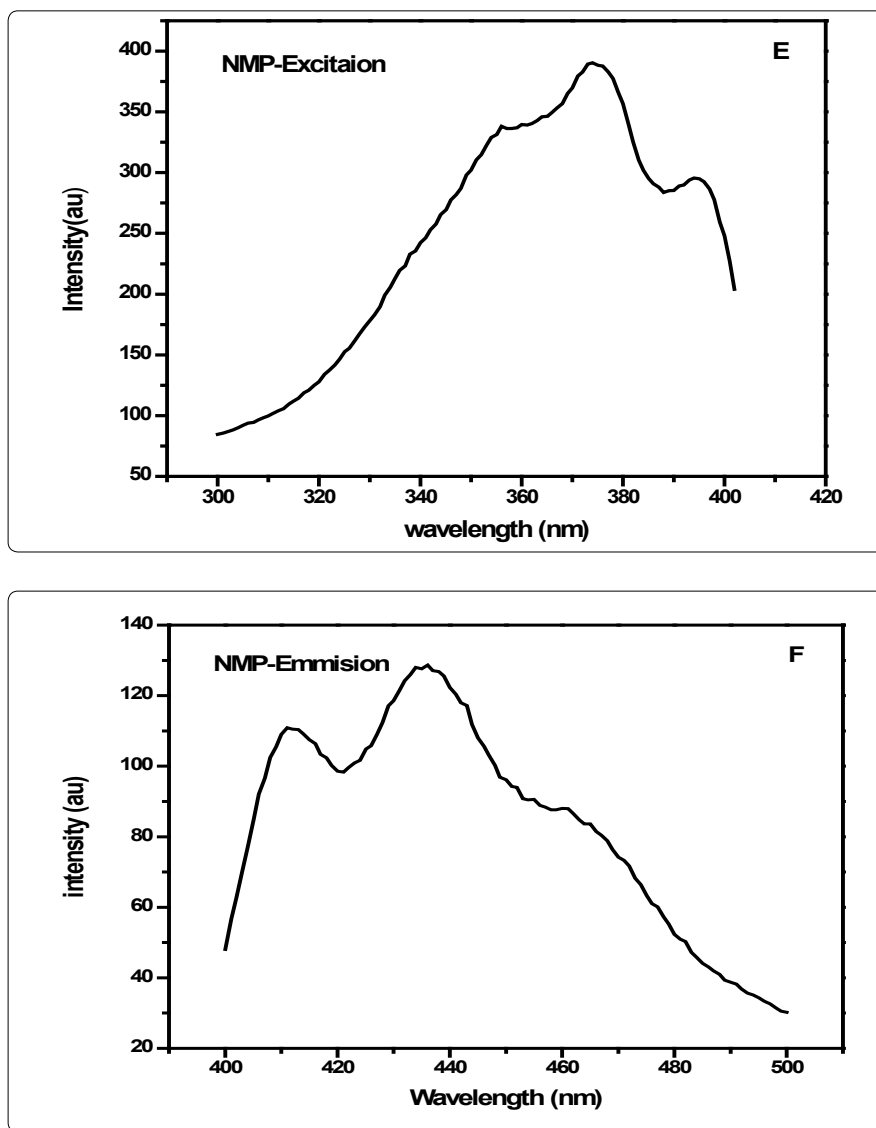

Figure 1. Fluoroscence excitation and emission of CSA-PANi in different solvents

$A$ and $B$ Excitation \& Emission spectra of CSA-PANi in solvent DMF respectively.

C and D Excitation \& Emission spectra of CSA-PANi in solvent DMSO respectively.

$\mathrm{E}$ and $\mathrm{F}$ Excitation \& Emission spectra of CSA-PANi in solvent NMP respectively

\section{Results and Discussion}

A variety of environmental factors affect fluorescence emission, including solvent polarity, inorganic and organic compounds, temperature, $\mathrm{pH}$, and the localized concentration of the fluorescent species. The high degree of sensitivity in fluorescence is primarily due to interactions that occur in the local environment during the excited state lifetime. A fluorophore can be considered an entirely different molecule in the excited state (than in the ground state), and thus will display an alternate set of properties in regard to interactions with the environment.Polar solvent molecules surrounding fluorophore interact with the dipole moment of the fluorophore to yield an ordered distribution of solvent molecules around the fluorophore?.

The fluorescence emission CSA-PANi occurs in the range from $409 \mathrm{~nm}$ to $411 \mathrm{~nm}$ with maximum emission at $418 \mathrm{~nm}$ in DMSO [10]. The fluorescence emission spectra are shown in the figure 1.The emission peak of the compound exhibit a gradual shift from DMF to NMP.

From the table it is observed that as the polarity of the solvent increases, there is a spectral shift which increases with increase in the polarity of the solvent as shown in the figure-2. The emission peak wavelength of CSA-PANi in DMF is $409 \mathrm{~nm}$ but in DMSO it is $418 \mathrm{~nm}$. This shift could be explained using solvent relaxation as shown in the figure- 2 .

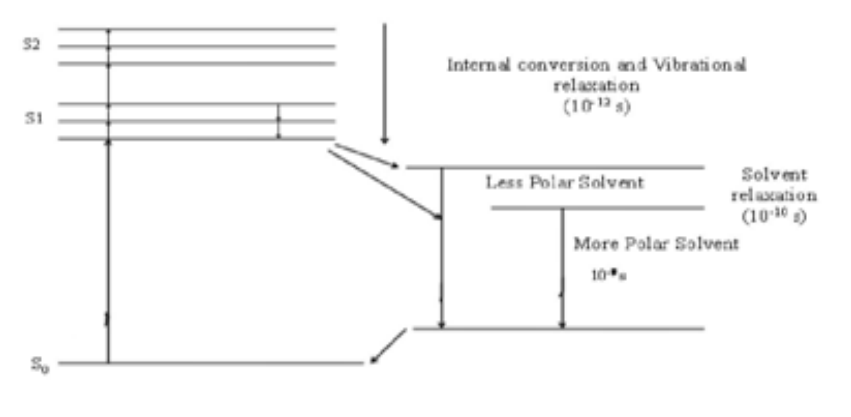

Figure 2. Schematics of solvent relaxation.

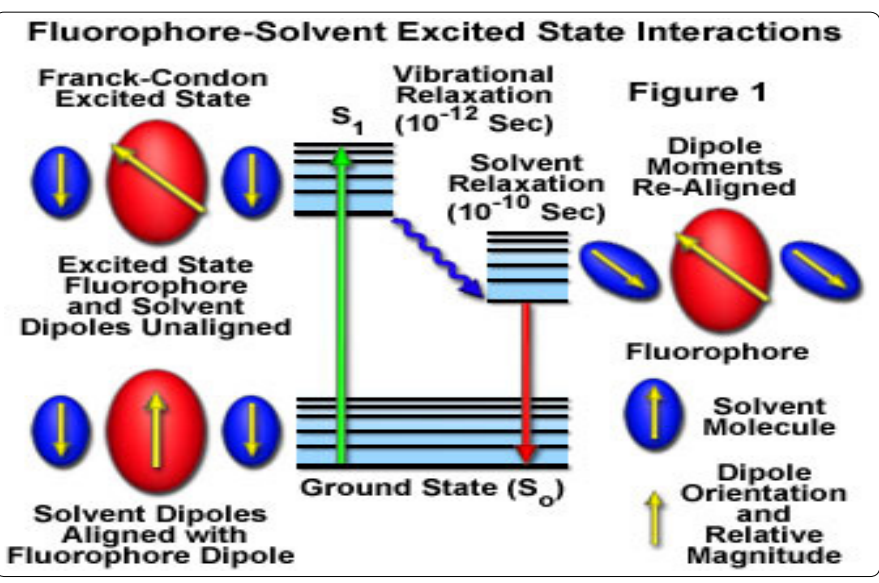

Figure 3. Fluorophore -solute excited state interactions 10

\section{Fluoropore - Solvent Interactions}

In a polymer solution, the fluorophore in the ground state is surrounded by polar solvent molecules having dipole moments that can interact with the dipole moment of the fluorophore to yield an ordered distribution of solvent molecules around the fluorophore [11]. By the absorption of energy fluorophore is excited to higher energy level and there will be change in the dipole moment which ultimately induces a rearrangement or reorientation of surrounding solvent molecules around the fluorophore which lowers the energy of excited state of fluorophore. This is called solvent relaxation. Solvent molecules will reduce the energy gap between the ground state and excited state of the fluorophore which results in red shift or Bathochromic shift of the fluorescence emission. Increasing the solvent polarity produces a correspondingly larger reduction in the energy level of the excited state [12]. Fluorescent molecules have larger dipole moment in excited state than in the ground state. Therefore different solvents having different polarity may differently affect the energies of ground state and excited state for a molecule. In non-polar solvent like xylene the excited state of the fluorophore is not stabilized and the energy gap between the ground state and excited state of the molecule increases. Thus the emission peaks are shifted to shorter wavelength or blue shift is observed [13].

The Solubility of CSA-PANi is found to be dependent on the polarity of the solvent. The solubility is high in the highly polar solvents selected and decreases as the polarity of the solvent decreases. 


\section{Conclusion}

The fluorescence data of CSA-PANi reveal that the nature and amount of shifts depend on the polarity of the solvents. It is concluded from the data that as the polarity of the solvent increases, CSA-PANi shows a bathochromic shift for $n-\pi$ and $\pi-\pi^{*}$ transitions. Knowledge about the excited electronic state dipole moments of the solute molecules is quite useful in designing nonlinear materials and in elucidation of the nature of the excited state.

\section{Acknowledgements}

The author Lakshmidevi V. acknowledges Government first grade college, Ramanagara, Karnataka, India for supporting throughout research work.

\section{References}

1. Jonas ad Silva M, Sanches AO, Malmonge LF, Malmong JA. Electrical, mechanical, and thermal analysis of natural rubber/Polyaniline-Dbsa composite. Mat. Res. 2014; 17(1). doi: 10.1590/S1516-14392014005000065

2. Alizadeh N, Akbarinejad A, Ghoorchian A. Photophysical Diversity of Water-Soluble Fluorescent Conjugated Polymers Induced by Surfactant Stabilizers for Rapid and Highly Selective Determination of 2, 4, 6-Trinitrotoluene Traces. ACS Appl. Mater. Interfaces. 2016; 8(37): 2490124908. doi: 10.1021/acsami.6b08577

3. Gong J, Yu J, Chen Y, Qu L. Gas-Solid phase method to synthesize polyaniline doped with heteropoly acid. Mater. Lett. 2002; 57(3): 765-770. doi: 10.1016/S0167-577X(02)00869-8

4. Cao Y, Smith P, Heeger AJ. Counter-ion induced processibility of conducting Polyaniline and of conducting polyblends of Polyaniline in bulk polymers. Synth Met. 1992; 48(1): 91-97. doi: 10.1016/0379-6779(92)90053-L
5. Homocianu1 $M$, Airine A, Dorohoi DO. Solvent Effects on the Electronic Absorption and Fluorescence Spectra. Journal of Advanced Research in Physics. 2011; 2(1): 011105.

6. Chen S, Li X, Ma H. New Approach for Local Structure Analysis of the Tyrosine Domain in Proteins by Using a Site-Specific and PolaritySensitive Fluorescent Probe. Chem Bio Chem. 2009; 10(7): 1200-1207. doi: 10.1002/cbic.200900003

7. Nandy Ritesh, Sankararaman S. Donor-acceptor substituted phenylethynyltriphenylene- excited state intramolecular charge transfer, solvatochromic absorption and fluorescence emission. Beilstein J. Org. Chem. 2010; 6: 992-1001. doi:10.3762/bjoc.6.112

8. Sakurovs R, Ghiggino KP. Solvent effects on the fluorescence of coumaric acids. Journal of photochemistry. 1983; 22(4): 373-377. doi: 10.1016/00472670(83)85016-3

9. Olympous Microscopy Resource Centre. Florida University.

10. Gulseven Sıdır Y, Sıdır I. Solvent effect on the absorption and fluorescence spectra of 7-acetoxy-6-(2, 3-dibromopropyl)-4, 8-dimethylcoumarin. Determination of ground and excited state dipole moment. Spectrochimica Acta Part A. Molecular and Biomolecular Spectroscopy. 2013; 102: 286-296. doi: 10.1016/j.saa.2012.10.018

11. Sakurovs R, Ghiggino KP. Solvent effects on the fluorescence of coumaric acids. Journal of photochemistry. 1983; 22(4): 373-377. doi: 10.1016/00472670(83)85016-3

12. Hrdlovic P, Donovalova J, Stankovicova H, Gaplovsky A. Influence of Polarity of Solvents on the Spectral Properties of Bichromophoric Coumarins. Molecules. 2010; 15(12): 8915-8932. doi: 10.3390/molecules15128915

13. Haidekker MA, Brady TP, Lichlyter D, Theodorakis EA. Effects of solvent polarity and solvent viscosity on the fluorescent properties of molecular rotors and related probes. Bioorg Chem. 2005; 33(6): 415-425. doi: 10.1016/j.bioorg.2005.07.005 